\title{
Chronic focal polymyositis in the adult
}

\author{
N E BHARUCHA AND J A MORGAN-HUGHES \\ From the Institute of Neurology, Queen Square, London
}

SUMMARY Four patients with chronic focal polymyositis are described. Treatment with steroids appeared to halt clinical progression. The clinical features in all four cases were so strikingly similar as to constitute a syndrome which can be mistaken for muscular dystrophy or spinal muscular atrophy unless investigated fully.

Uncomplicated polymyositis is an inflammatory disease of muscle of uncertain aetiology, which typically presents with symmetrical limb girdle weakness, usually without wasting or reflex change. ${ }^{1}$ However, there have been occasional reports in the literature describing unusual presentations. These have included predominantly distal rather than proximal muscle involvement, ${ }^{2-4}$ a fascioscapulohumeral distribution of weakness, closely mimicking FSH dystrophy ${ }^{5-7}$ and weakness and wasting largely confined to the quadriceps muscles. ${ }^{8}$ Highly selective involvement of individual muscles or groups of muscles, however, is unusual in polymyositis and when present is generally regarded to be more in favour of a muscular dystrophy. Walton and Adams $^{9}$ found only two patients with selective wasting and weakness in their series of over 40 cases.

This paper describes four adult patients with morphologically proven chronic polymyositis, who exhibited highly selective muscle wasting and weakness, which remained largely confined to the forearm flexors, brachio radialis and quadriceps muscles.

\section{Case histories}

Case 1 (Nat Hosp No A82412)

A 56-year-old housewife was first admitted to the National Hospital in June 1975, with a ten-year history of slowly progressive weakness of the legs, characterised by difficulty in climbing or descending stairs, and in getting out of a low chair. There was no history of dysphagia, muscle or joint pain, or skin rash, and her general health had been good. In infancy she had suffered from acute poliomyelitis and had been left with considerable wasting, weakness and shortening of the left arm. There was no family history of neuro-muscular disease and no known consanguinity. On examination her gait was normal but

Address for reprint requests: Dr JA Morgan-Hughes, The National Hospital for Nervous Diseases, Queen Square, London WC1N 3BG. Accepted 28 February 1981 she was unable to climb stairs without using her arms and when descending stairs her legs would tend to buckle at the knees. She was able to sit up from a supine position without difficulty. The cranial nerves were normal except for minimal weakness of neck flexion. In the upper limbs there was long standing wasting and weakness and shortening of the left arm, due to old poliomyelitis. Muscle power in the right arm was normal. In the lower limbs there was marked wasting and weakness of both quadriceps muscles (fig 1). The other lower limb muscles were normal in both bulk and strength. The limb reflexes were present, but depressed (except in the left arm, where they were absent) and the plantar responses were flexor. Sensation was normal to all modalities.

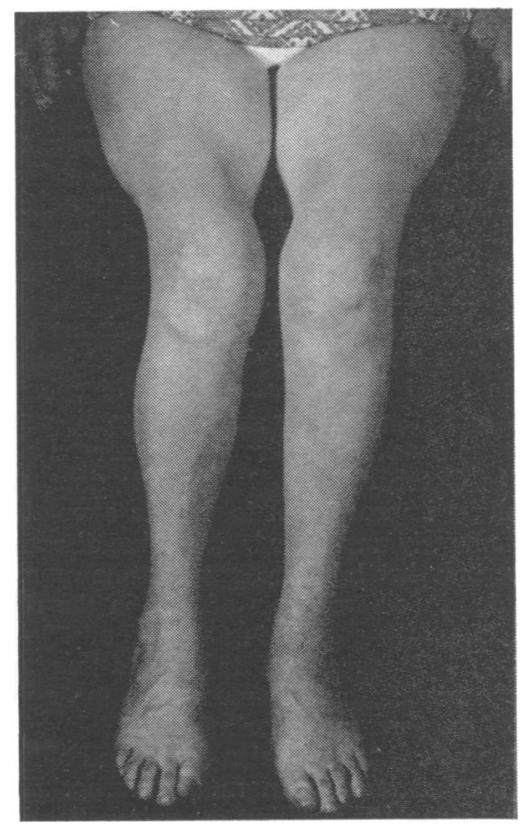

Fig 1 Case 1. Showing marked bilateral selective wasting of the quadriceps. 


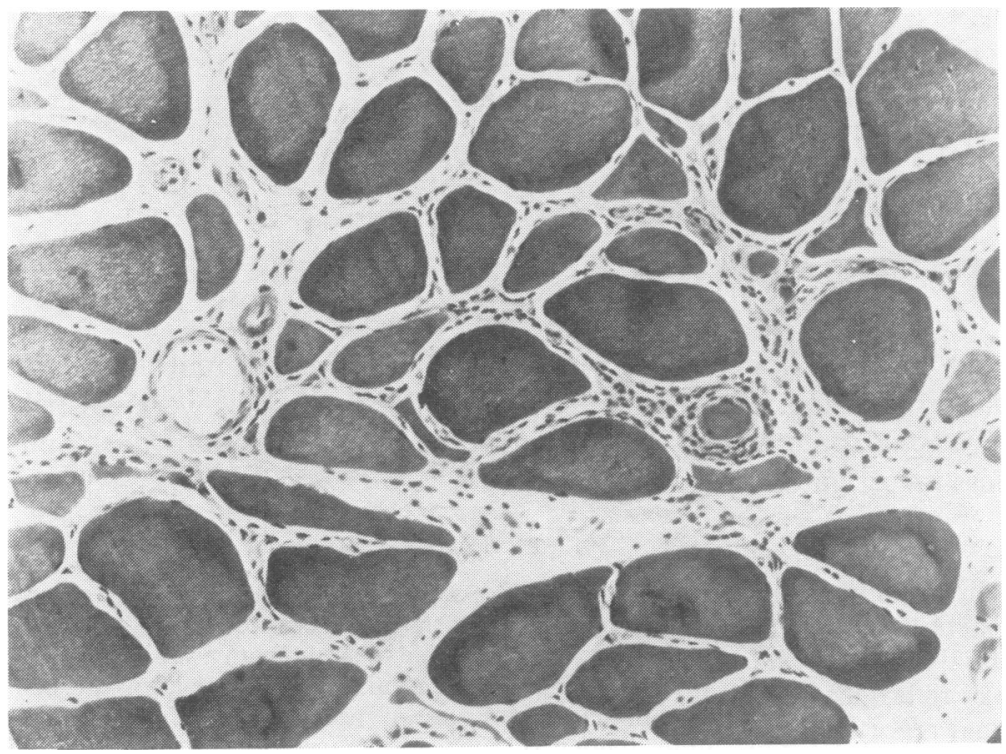

Fig 2 Case 1. Muscle biopsy $(\times 100)$ of right triceps muscle showing moderately variable muscle fibre size, small collections of interstitial and perivascular inflammatory cells and occasional regenerating muscle fibres.

Her blood pressure was $120 / 70 \mathrm{~mm} / \mathrm{Hg}$. The following investigations were normal; haemoglobin, full blood count, ESR, serology, serum electrolytes and blood urea, serum calcium, phosphorous and alkaline phosphatase blood glucose, liver function tests, T4, plasma, cortisol, chest radiograph and ECG. An auto-antibody screen was negative. The serum CPK was 128 IU/1 (normal 10-43).

Electromyographic sampling of the right biceps, right vastus medialis and right tibialis anterior muscles showed profuse fibrillation potentials at rest. The interference pattern was full, but there was an excess of short duration polyphasic motor unit action potentials of low amplitude indicating a primary muscle disease. Biopsy of the right triceps muscle showed moderate variation in muscle fibre size, with small collections of inflammatory cells, which were mainly interstitial but sometimes perivascular (fig 2). Occasional regenerating muscle fibres were also seen.

The patient was treated with prednisone $60 \mathrm{mg}$ on alternate days and after two months her serum CPK had fallen to $31 \mathrm{IU} / \mathrm{l}$. Because of mild epigastric discomfort and the appearance of small subcapsular cataracts the dose of prednisone was reduced and finally stopped in October 1978. During this time, there had been a slight improvement in muscle strength but the signs were largely unchanged. Following discontinuation of the steroids her leg weakness became more marked and she developed weakness of grip in the right hand and difficulty with swallowing solid foods. When seen in February 1979, there was definite wasting and weakness of the brachio-radialis muscle on the right (fig 3) and also of the forearm flexor muscles. The weakness of the quadriceps muscles had also progressed but the other lower limb muscles were normally strong. The serum CPK had risen to $174 \mathrm{IU} / 1$ but other investigations were normal. Prednisone, $20 \mathrm{mg}$ on alternate days was recommenced with considerable improvement in her mild

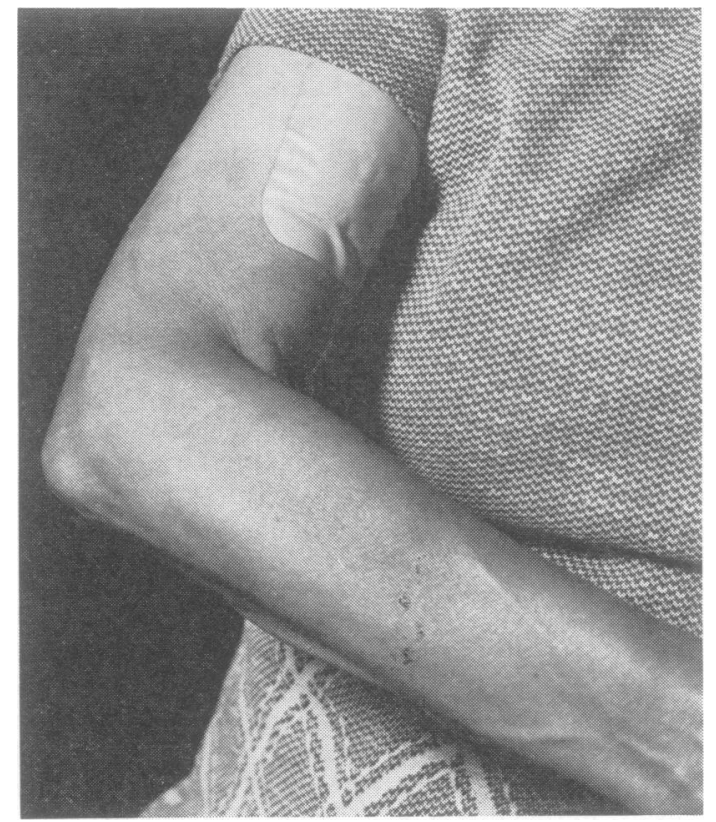

Fig 3 Case 1. To show wasting of right brachioradialis.

dysphagia, but no significant change in the limb weakness. The serum CPK fell to $33 \mathrm{IU} / 1$. When last seen in August 1979, she was taking $30 \mathrm{mg}$ of prednisone on alternate days and her condition was static.

Case 2 (Nat Hosp No A97403)

A 53-year-old man was admitted to the National Hospital in May 1978, with a six-year history of slowly progressing weakness of the legs and of his grip. He had difficulty in 
rising from a chair and in climbing stairs and when descending stairs his legs tended to buckle at the knees. $\mathrm{He}$ also complained of weakness of grip with difficulty in holding a briefcase or a golf club. There was no history of muscle pain, dysphagia or skin rash, and the family history was negative. On examination, his gait was normal but he was unable to climb stairs without using his arms and descended stairs backwards, locking his knees at each step to prevent buckling. The cranial nerves were normal except for longstanding weakness of the left frontalis and orbicularis oculi muscles. In the upper limbs there was severe wasting and weakness of the brachioradialis muscles and the long finger flexors (fig 4). The other muscles in the arms were of normal bulk and strength. In the legs there was gross wasting and weakness of both quadriceps muscles, with sparing of the other muscle groups (fig 5). The limb reflexes were depressed and the knee jerks were only obtained with reinforcement. The plantar responses were flexor and sensory examination was normal. General examination was also normal with a blood pressure of $140 / 90 \mathrm{mmHg}$. The following investigations were normal; haemoglobin, full blood count, ESR, serology, serum electrolytes and blood urea, serum calcium, inorganic phosphorous, and alkaline phosphatase, blood glucose, liver function tests, T4, chest radiograph and ECG. An auto-antibody
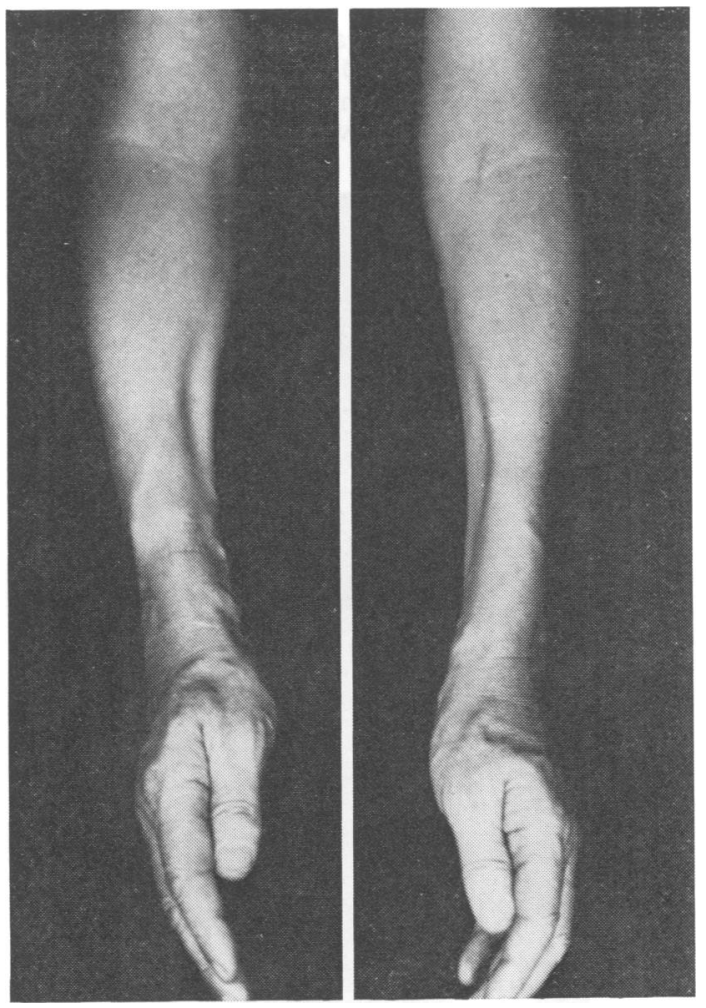

Fig 4 Case 2. Showing advanced wasting of forearm flexors.

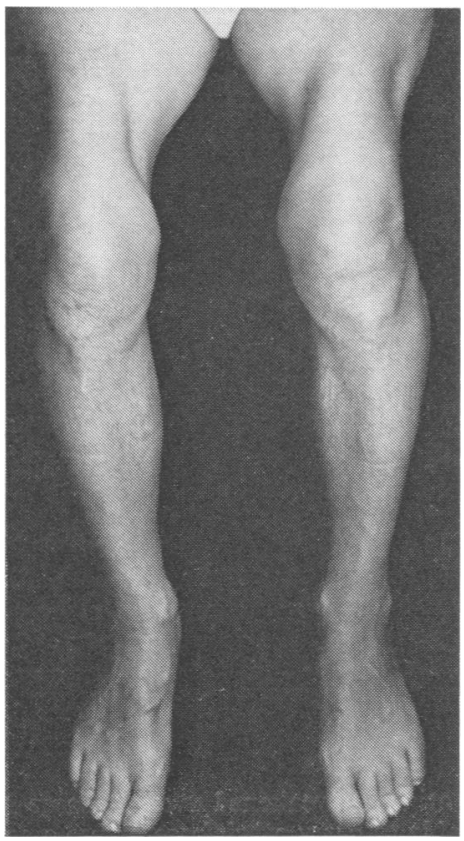

Fig 5 Case 2. Showing gross bilateral and selective quadriceps wasting.

screen was negative. The serum CPK was 96 IU/1. (normal 10-66).

Electromyography showed profuse fibrillation in the right biceps and brachioradialis muscles, with increased insertion activity in the right tibialis anterior. All the muscles sampled showed short duration low amplitude polyphasic motor unit action potentials indicating primary muscle disease. Motor and sensory conduction in the right median, ulnar and sural nerves was normal. A biopsy of the left biceps muscle showed marked variation in muscle fibre size with a number of hypertrophied fibres, which showed early splitting. There were large collections of inflammatory cells between the muscle fibres and around the blood vessels (fig 6). Some of the fibres showed vacuolar changes. A number of regenerating fibres were present.

The patient was treated with prednisone $80 \mathrm{mg}$ on alternate days and during the next two months there was some improvement in muscle strength. The serum CPK fell to $25 \mathrm{IU} / 1$ after five weeks of treatment. The steroid dosage was gradually reduced and when last seen in April 1980, the CPK was $33 \mathrm{IU} / 1$ and his condition was relatively static. He was taking $30 \mathrm{mg}$ of prednisone on alternate days.

Case 3 (Nat Hosp No A76641)

A 54-year-old man was first admitted to the National Hospital in May 1974, with a two-year history of weakness involving the legs and hands. His legs tended to buckle at the knees when descending stairs and he had difficulty in getting out of a low chair and in climbing 


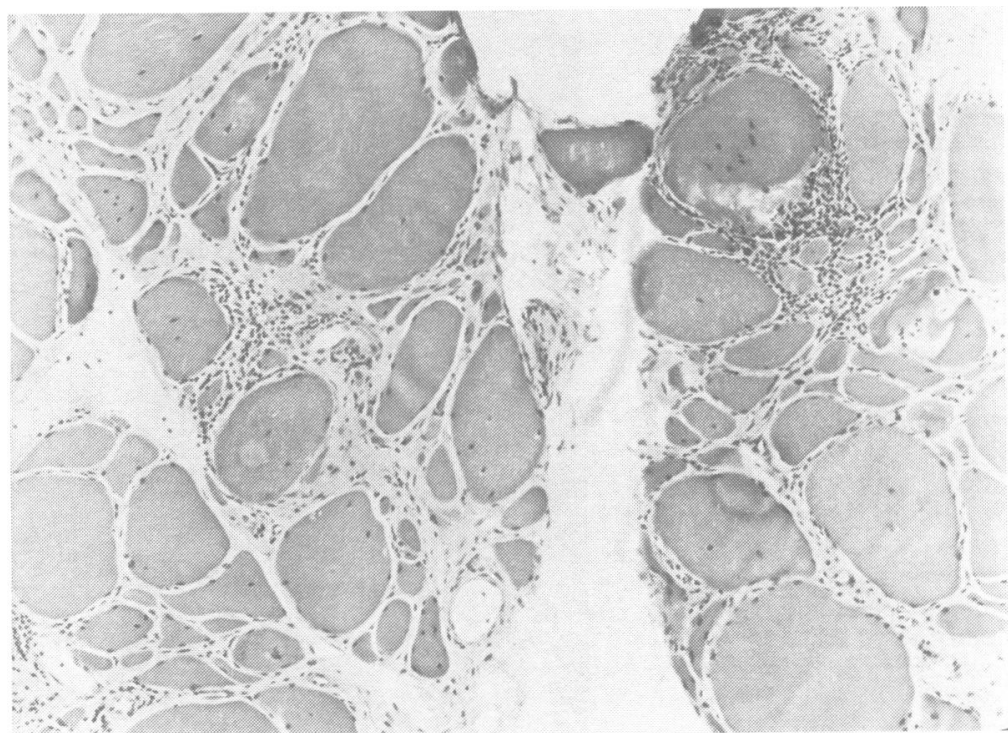

Fig 6 Case 2. Muscle biopsy ( $\times 67)$ showing variable muscle fibre size, some hypertrophied fibres and some evidence of regeneration. Note the large collections of inflammatory cells between fibres and around blood vessels. stairs. His grip had weakened over the same period and he was unable to carry a briefcase or use a screwdriver. There was no other significant history and his previous health had been good. On examination, his gait was normal but he was unable to rise from a chair or climb steps without using his arms. The cranial nerves were normal. In the upper limbs, the long finger flexors in the forearm were markedly wasted and weak. The muscle bulk and strength elsewhere in the upper limbs was normal. In the legs there was selective wasting and weakness of both quadriceps muscles (fig 7) the other lower limb muscles being normal. The limb reflexes were normal and there were no sensory changes. The following investigations were normal; haemoglobin, full blood count, serology, serum electrolytes and blood urea, serum calcium, phosphorous and alkaline phosphatase, blood sugar, liver function tests, T4, chest radiograph and ECG. The ESR was $35 \mathrm{~mm}$ in the hour and the ANF was positive in a titre of less than one in ten. The serum CPK was $250 \mathrm{IU} / 1$.

Electromyographic sampling of the right rectus femoris, the right flexor carpi ulnaris and the right biceps showed spontaneous fibrillation potentials with polyphasic motor unit action potentials, indicating primary muscle disease. Motor and sensory conduction velocities in the right median and ulnar nerves were normal. Muscle biopsy (fig 8 ) showed marked variation in muscle fibre size, with large collections of inflammatory cells which were interstitial and perivascular in distribution. A number of muscle fibres showed evidence of necrosis with phagocytosis and regenerating fibres were common.

Treatment was commenced with prednisone $80 \mathrm{mg}$ on alternate days, and during the next six weeks there was improvement in muscle strength. He was by now able to rise from a chair without using his arms, although he still had difficulty in climbing and descending stairs.
The serum CPK fell to $9 \mathrm{IU} / 1$. The dose of prednisone was gradually reduced to $20 \mathrm{mg}$ on alternate days and on this regime the weakness in his legs became worse. Progression of the disease was not halted by increasing the dose of prednisone to $60 \mathrm{mg}$ on alternate days and because of the development of cushingoid features, azathioprine, $200 \mathrm{mg}$ a day was added to his treatment

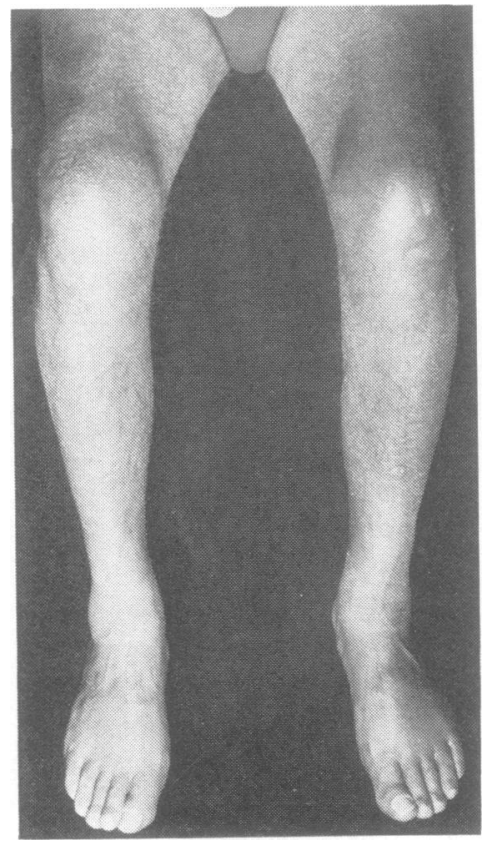

Fig 7 Case 3. Bilateral selective quadriceps wasting. 


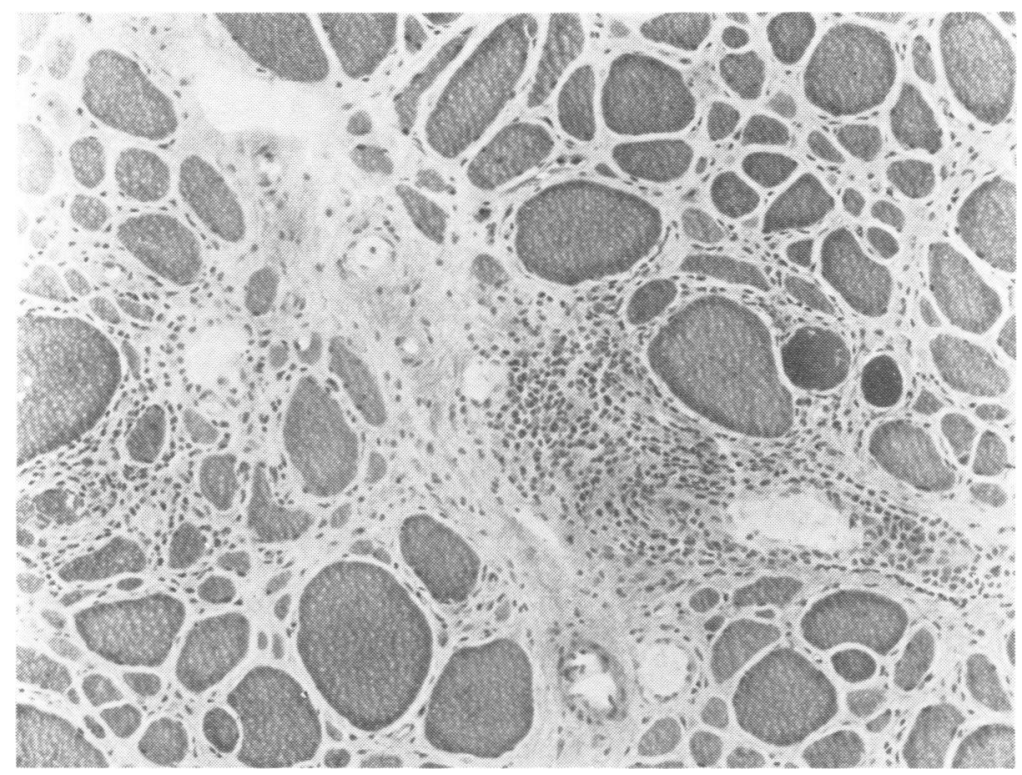

Fig 8 Case 3. Muscle biopsy $(\times 100)$ shows variable fibres size, inflammatory infiltrates, muscle necrosis and phagocytosis with some evidence of regeneration.

in April, 1978. The prednisone was gradually withdrawn but because of further slow progression in his weakness it was recommenced in October 1979. When last seen in March 1980, he was taking azathioprine, $200 \mathrm{mg}$ per day together with prednisone, $20 \mathrm{mg}$ daily, and his condition was relatively static.

\section{Case 4}

This 65-year-old patient was first seen at the National Hospital in July 1972, with a two-year history of progressive weakness of the arms and legs. On examination, he was unable to rise from a chair or step up a steep step without using his arms. The cranial nerves were normal, but in the limbs he had selective wasting and weakness, which was confined to the brachio-radialis muscles and the forearm flexor muscles in the arms and the quadriceps muscles in the legs. The limb reflexes were depressed but present, and sensation was normal. The following investigations were normal; haemoglobin, full blood count, ESR, serology, serum electrolytes and blood urea, serum calcium, phosphorus and alkaline phosphatase, blood sugar, liver function tests, T4, chest radiograph and ECG. An auto-antibody screen was negative. The serum CPK was $106 \mathrm{IU} / 1$. Electromyographic sampling of the right triceps and tibialis anterior muscles showed no spontaneous activity, but the motor units were polyphasic and of brief amplitude, indicating primary muscle disease. A muscle biopsy showed marked variation in muscle fibre size with large collections of inflammatory cells which were mainly interstitial but sometimes perivascular in distribution (fig 7). Regenerating fibres were relatively common.

The patient was started on prednisone, $80 \mathrm{mg}$ on alternate days, and there was considerable initial im-

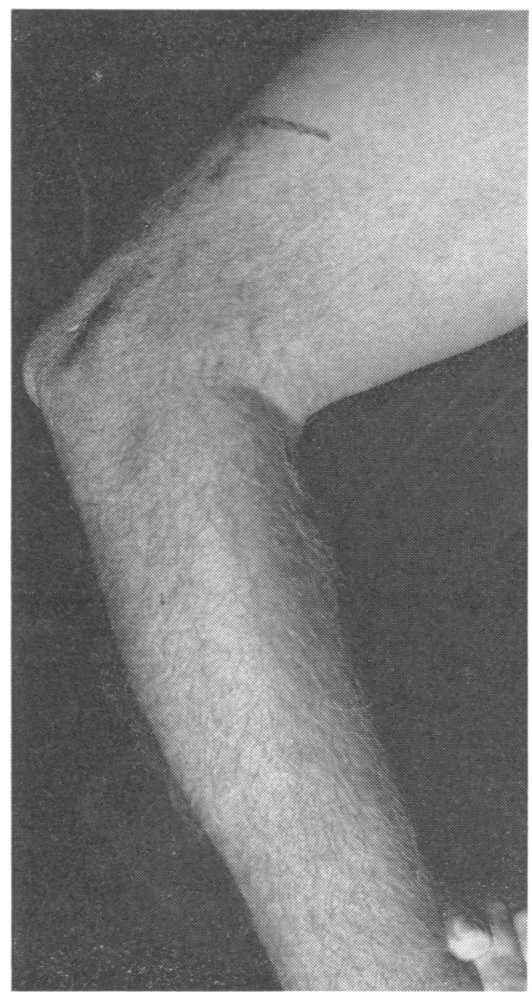

Fig 9 Case 4. To show wasting of right bronchioradialis. 


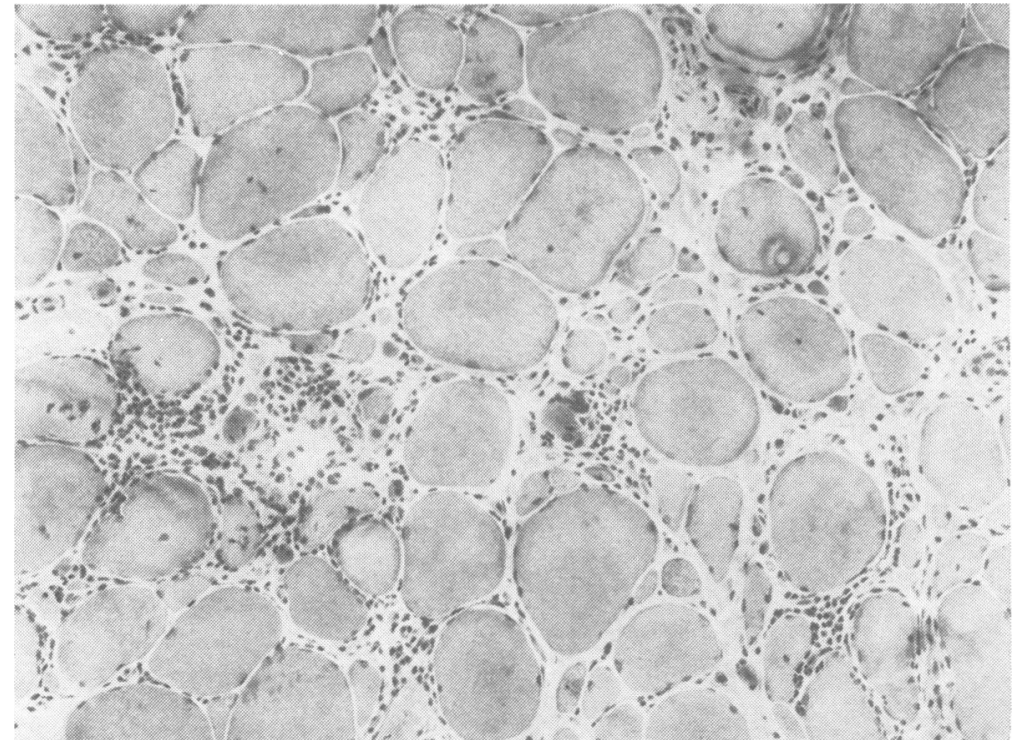

Fig 10 Case 4. Muscle biopsy $(\times 80)$ shows variable fibre size, mainly interstitial inflammatory cells and regenerating fibres. provement in muscle strength during the next three to six months. The CPK fell to $50 \mathrm{IU} / \mathrm{l}$. His improvement was maintained during the next two years, on a maintenance dose of prednisone (between 17.5 and $20 \mathrm{mg}$ on alternate days), but then the disease began to progress. The prednisone was increased up to $60 \mathrm{mg}$ on alternate days without further improvement. Both cyclophosphamide and azathioprine were added, but because of a severe leucopenia these drugs were withdrawn. When last seen in December, 1977, the disease was relatively static and his serum CPK was 95 IU/1.

\section{Discussion}

These patients were suffering from a very chronic form of inflammatory myopathy. In each case the onset was insidious and the wasting and weakness was largely confined to the quadriceps muscles, the forearm flexors and the brachio-radialis. The laboratory findings, which included elevated CPK levels, myopathic potentials with fibrillations (in three out of the four cases) and collections of inflammatory cells on muscle biopsy were all compatible with the diagnosis of chronic polymyositis. All four patients also showed some initial improvement on high doses of steroids which was accompanied by a gradual reduction in the CPK levels in the blood. Although they were on a low dose of prednisone the weakness progressed a little. It did not appear to spread to involve other muscles and all four patients have remained ambulant and only moderately disabled over prolonged periods of follow-up.

This group of middle-aged patients with chronic polymyositis had such a strikingly similar distribution of muscle weakness as to constitute a syndrome. They had no evidence of associated malignancy or collagen vascular disease and have been followed up for periods ranging from 6-14 years. Clinically this picture is most closely resembled by late onset muscular dystrophy ${ }^{10-12}$ and spinal muscular atrophy. However, none of these patients had a family history of neuromuscular disease or a history of cosanguinity. Moreover, the absence of muscular hypertrophy or fasciculation and the preservation of the reflexes in the presence of severe wasting and weakness would be much more in favour of an inflammatory myopathy. This was confirmed by the presence of profuse fibrillation on EMG (in three out of four cases,) muscle biopsy appearance, the clinical response to steroids and lowering of CPK levels during treatment with steroids. It is interesting that throughout prolonged follow-up the disease showed little or no evidence of spread to other muscles. Although steroids proved ineffective in improving the strength of severely wasted muscles they may have prevented the disease from spreading as evidenced by Case 1 who deteriorated rapidly when treatment was discontinued.

\section{References}

1 Bohan A, Peter JB, Bowman RL, Pearson CM. A computer-assisted analysis of 153 patients with polymyositis and dermatomyositis. Medicine (Baltimore) 1977 ; 56:255-86.

2 Eaton LM. Perspective of neurology in regard to 
polymyositis. A study of 41 cases. Neurology (Minneap) 1954; 4:245-63.

3 Hollinrake $K$. Polymyositis presenting as distal muscle weakness. A case report. J Neurol Sci 1969; 8:479-84.

4. Van Kasteren BJ. Polymyositis presenting with chronic progressive distal muscular weakness. $J$ Neurol Sci 1979; 41:307-10.

5 Rothstein TL, Carlson CB, Sumi SM. Polymyositis with facioscapulohumeral distribution. Arch Neurol 1971; 25:313-9.

6 Munsat TL, Piper D, Cancilla P, and Mednick J. Inflammatory myopathy with facioscapulohumeral distribution. Neurology (Minneap) 1972; 22:335-47.

7 Bates D, Stevens JC, Hudgson P. "Polymyositis" with involvement of facial and distal musculature. One form of the facioscapulohumeral syndrome?
J Neurol Scis, 1973; 19:105-8.

8 Mohr PD, Knowlson TG. Quadriceps myositis: an appraisal of the diagnostic criteria of quadriceps myopathy. Postg Med J 1977; 53:757-60.

9 Walton JN, Adams RD. Polymyositis. Edinburgh: Livingstone, 1958.

10 Shy GM, McEachern D. The Clinical features and response to cortisone of menopausal muscular dystrophy. J Neurol Neurosurg Psychiatry 1951; 101-7.

11 Adams RD, Denny-Brown D, Pearson CM. Diseases of muscle. New York; PB Hoeber Inc., 1953.

12 Nevin S. Two cases of muscular degeneration occurring in late adult life, with a review of the recorded cases of late progressive muscular dystrophy (late progressive myopathy). $Q J \mathrm{Med}$ (New Series) 1936; 5:51-68. 